\title{
Research on vision stabilization system of mobile platform
}

\author{
Miao Yangyang ${ }^{a}$, Gu Juping ${ }^{b}, X$ Yiming $^{c}$, Yuan Linlin ${ }^{d}$, Qiu Tianbo, Li Pingwen \\ School of Electrical Engineering, Nantong University, Nantong, Jiangsu Province, China \\ ad80184167@qq.com, bgu.jp@ntu.edu.cn, cyimingx@ntu.edu.cn, d935600148@qq.com
}

Keywords: mechanical image stabilization; electronic image stabilization; gray projection algorithm; motion estimation

\begin{abstract}
Aiming at the problem that the large flutter of mobile platform makes the camera screen blur, a compound image stabilization system was designed which includes mechanical and electronic image stabilization. In this system, the mechanical part is used to compensate the deflection, which can maintain the elementary stability of the camera. At the same time, the electronic part based on sub-block gray projection algorithm can maintain the stability of video images and effectively restrain the interference of local moving objects such as vehicles in the pictures. The experimental results show that the average PSNR of video images is raised $16 \mathrm{~dB}$ after stabilization and the system can meet the real-time requirement.
\end{abstract}

\section{Introduction}

The working environment of mobile platform is out of control in many cases, and the camera system on the platform is inevitably influenced by the motion and vibration of carriers. This makes the display image shaking and fuzzy. So the image stabilization processing is necessary. On one hand, people can achieve better image details and feel comfortable with the perception of observation by the stable video, which contributes to the artificial observation and cognition. On the other hand, image stabilization can also be used as the preprocessing of subsequent processing which includes identification, tracking and compression coding.

Image stabilization technology includes optical image stabilization, mechanical image stabilization and electronic image stabilization [1]. The precision of optical one is high, but the cost is relatively high. The accuracy of mechanical one is closely related to the hardware cost. In [2], the stability and control methods of some new-type of mechanical stabilization platforms are analyzed, and their applications in the military field are described. The electronic one is widely used because of the advantages such as easy operation, high flexibility, etc [3]. The electronic image stabilization also includes the block matching, the feature matching [4-5], and the gray projection. The gray projection method estimates the motion vector by the whole gray distribution of the image, which can reduce the calculation quantity while ensuring certain accuracy[6]. In recent years, many scholars have improved the gray projection method and made some progress. In [7], the improved circular projection algorithm is used to reduce the interference of the moving foreground to the motion estimation by using the adaptive block based on the bit plane of Pyramid. In [8], the accuracy and real-time performance of motion estimation are improved by screening the sub regions according to the gray gradient. However, the above-mentioned literatures do not solve the problem of large-scale migration. In view of the problems of large-scale flutter of mobile platform and the interference of moving foreground, this paper proposed a compound image stabilization method. The method achieves coarse control by mechanical image stabilization and fine control by electronic one, and finally realizes good stabilization under complex background.

\section{Mechanical image stabilization based on MPU6050}

In the course of operation, the large-amplitude jitter of the mobile platform is easy to occur due to the bumps of the road surface. The front frame of camera system often goes out of the camera's field of view, which will make two adjacent frames irrelevant, as shown in Fig.1. In this case, the electronic 
image stabilization method is no longer applicable. Mechanical image stabilization method must be applied.

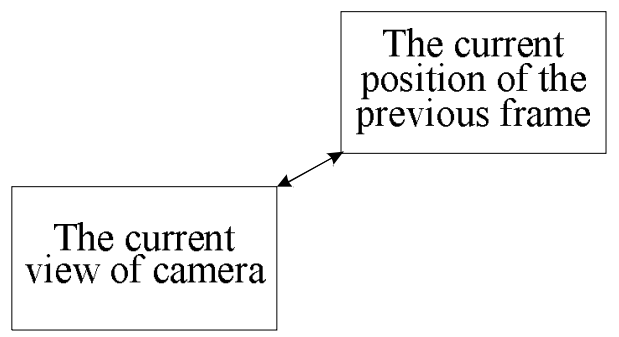

Fig.1 Large-amplitude jitter of camera system

At present, the method of mechanical image stabilization is mostly used in military field. The method usually requires high-precision fiber optic gyro (FOG) and servo motor to realize image stabilization. And the system is quite expensive. In this paper, a low-cost method of mechanical image stabilization was designed in order to ensure the basic stability of camera system.

\section{Hardware design of mechanical image stabilization}

The overall structure of mechanical image stabilization is shown in Fig.2. It mainly includes the master control module of MSP430, the angle acquisition module of MPU6050 and the angle compensation module of stepping motor.

\begin{tabular}{|c|c|}
$\begin{array}{c}\text { Angle acquisition } \\
\text { module of MPU6050 }\end{array}$
\end{tabular}$\Rightarrow$\begin{tabular}{c}
$\begin{array}{c}\text { Control module } \\
\text { of MSP430 }\end{array}$ \\
$\begin{array}{c}\text { Angle compensation } \\
\text { module of stepping motor }\end{array}$ \\
\hline
\end{tabular}

Fig.2 Overall structure of mechanical image stabilization

Angle acquisition module uses six-axis motion sensor MPU6050. It integrates three-axis MEMS gyroscope, three-axis MEMS accelerometer, and each axis corresponds to a 16-bit ADC. Among them, the measurable range of the gyroscope is $+250,+500,+1000,+500 \%$, and the measurable range of the accelerometer is $\pm 2, \pm 4, \pm 8, \pm 16 \mathrm{~g}$, which can measure the angle change of less than one degree [9]. MPU6050 also includes digital motion processing (DMP) engine, which can reduce the load of complex data fusion.

The two-phase and four-line stepping motor is selected in the angle acquisition module. The design of the step angle of about 1 degree can meet the requirements of rapidity and stability because the mechanical image stabilization will solve the problem of the large-angle deflection of camera.

\section{Software design of mechanical image stabilization}

In this paper, the data of the acceleration and the angular velocity of the gyroscope are processed by the quaternion algorithm, and Euler angle is obtained.

Define the quaternion $\mathrm{q}$ for a four-dimensional vector:

$$
\begin{aligned}
& q=\left[\begin{array}{llll}
q_{1} & q_{2} & q_{3} & q_{4}
\end{array}\right]^{T} \\
& q_{1}=\cos (\alpha / 2) \\
& q_{2}=e_{x} \sin (\alpha / 2) \\
& q_{3}=e_{y} \sin (\alpha / 2) \\
& q_{4}=e_{z} \sin (\alpha / 2)
\end{aligned}
$$

In Eq. $1, \alpha$ is the rotating Euler angle; $e_{x}, e_{y}$ and $e_{z}$ are respectively $\mathrm{X}, \mathrm{Y}$, and Z Euler axis.

According to the rotation order $\mathrm{Z} \rightarrow \mathrm{X} \rightarrow \mathrm{Y}$, the attitude matrix can be obtained by Euler angles and the quaternion. 


$$
\left\{\begin{array}{l}
\sin \varphi=2\left(q_{2} q_{3}+q_{1} q_{4}\right) \\
\cos \varphi \sin \theta=2\left(q_{2} q_{4}-q_{1} q_{3}\right) \\
\cos \varphi \cos \theta=q_{3}^{2}+q_{4}^{2}-q_{1}^{2}-q_{2}^{2} \\
\cos \varphi \sin \psi=2\left(q_{3} q_{4}-q_{1} q_{2}\right) \\
\cos \varphi \cos \psi=q_{2}^{2}+q_{4}^{2}-q_{1}^{2}-q_{3}^{2} .
\end{array}\right.
$$

In Eq.2, $\varphi$ is the roll angle; $\theta$ is the pitch angle; $\psi$ is the heading angle.

When Euler angles $\varphi, \theta, \psi \in\left(-\frac{\pi}{2}, \frac{\pi}{2}\right)$, the conversion relations between Euler angles and the quaternion are shown in Eq.3.

$$
\left\{\begin{array}{l}
\varphi=\arcsin \left[2\left(q_{2} q_{3}+q_{1} q_{4}\right)\right] \\
\theta=\arctan \frac{2\left(q_{2} q_{4}-q_{1} q_{3}\right)}{q_{3}^{2}+q_{4}^{2}-q_{1}^{2}-q_{2}^{2}} \\
\psi=\arctan \frac{2\left(q_{3} q_{4}-q_{1} q_{2}\right)}{q_{2}^{2}+q_{4}^{2}-q_{1}^{2}-q_{3}^{2}}
\end{array}\right.
$$

After calculating the deflection angle of the camera, the single-chip microcomputer MSP430 drives and controls stepping motor to compensate the angle so that the camera will maintain in a generally stable or slightly shaking state. Program flow chart is shown in Fig.3.

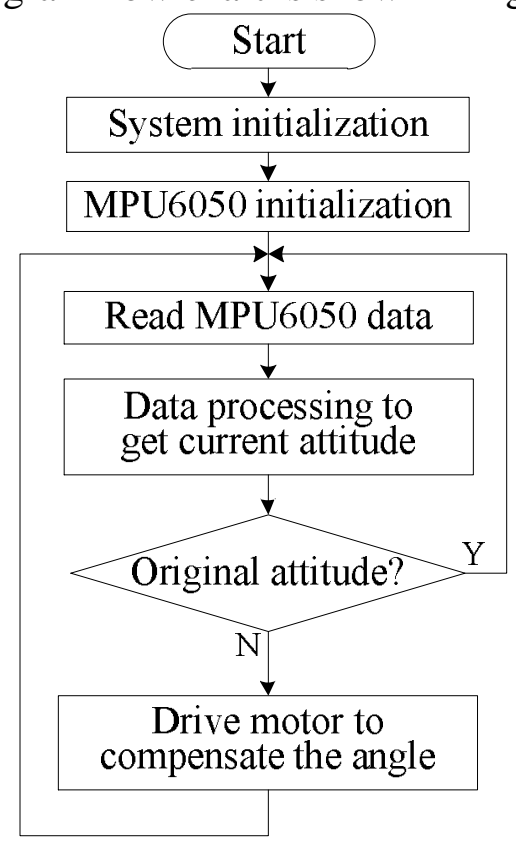

Fig.3 Program flow chart of the software design

\section{Electronic image stabilization based on sub-block gray projection}

After the processing of mechanical image stabilization, the video output of the camera system only exits some small, high frequency jitters. This paper conducts the second improvement, making use of electronic image stabilization based on sub-block gray projection.

Firstly, the method grays each frame in the video sequence. Then, the gray values in two horizontal and vertical directions are respectively mapped into one-dimensional wave. That is to say, one-dimensional gray information in two directions can represent two-dimensional image information[10-11]. The formula can be expressed as follows. 


$$
\left\{\begin{array}{l}
G_{\mathrm{n}}(j)=\sum_{i} G_{\mathrm{n}}(i, j) \\
G_{\mathrm{n}}(i)=\sum_{j} G_{\mathrm{n}}(i, j)
\end{array}\right.
$$

In Eq.4, $G_{\mathrm{n}}(i, j)$ stands for the gray value of the Nth frame image's pixel in coordinates $(i, j)$; $G_{\mathrm{n}}(j)$ means the superimposed gray value of the column $\mathrm{J}$ in the Nth frame; $G_{\mathrm{n}}(i)$ means the one of row I.

After gray projection curves of row and column are obtained, two curves in the current frame and the ones in the reference frame are calculated through cross correlation. The motion vector in the horizontal or vertical direction between the current frame and the previous frame is the only peak to peak value of the two curves. The correlation calculation is as follows.

$$
\left\{\begin{array}{l}
C\left(\omega_{H}\right)=\sum_{j=1}^{N-2 n}\left[G_{r}\left(j+\omega_{H}-1\right)-G_{n}(n+j)\right]^{2} \\
C\left(\omega_{v}\right)=\sum_{i=1}^{M-2 m}\left[G_{r}\left(i+\omega_{v}-1\right)-G_{n}(m+i)\right]^{2}
\end{array}\right.
$$

In Eq.5, $G_{\mathrm{r}}(j)$ and $G_{\mathrm{r}}(i)$ respectively represent the gray projection values of column $\mathrm{J}$ and row I in the reference frame image; $\mathrm{N}$ and $\mathrm{M}$ are the calculated numbers of columns and rows; $\mathrm{n}$ and $\mathrm{m}$ are the search width in the horizontal and vertical directions.

It assumed that $\omega_{H_{\min }}$ and $\omega_{v_{\min }}$ are respectively the minimum values of $\omega_{H}$ and $\omega_{V}$. Then, the motion vectors of the Nth frame image which is relative to the reference frame in the horizontal and vertical direction are as follows[12].

$$
\left\{\begin{array}{l}
\Delta x=n+1-\omega_{H_{\text {min }}} \\
\Delta y=m+1-\omega_{v_{\text {min }}}
\end{array}\right.
$$

The method of traditional gray projection can only detect the movement in the simple and fixed scenes when the camera system moves in the horizontal and vertical direction. However, there are a lot of moving vehicles such as pedestrians and other targets in the traffic scene, which is easy to interfere the motion estimation. In this paper, the method of sub-block gray projection is used to solve the problem.

\section{Selection of reference frame}

The appropriate selection of reference frame is the precondition of the accurate inter-frame motion estimation. Generally there are 2 ways to choose: 1) Fixed frame matching, which uses the first frame image as a reference frame and the subsequent images as the current frame; 2) Adjacent frame matching, which uses the first frame of two adjacent frame images as the reference frame and the second frame as the current frame.

There are a lot of moving objects in the traffic scenes, so the difference between the first frame image and subsequent frame are quite big. Thus, it's more appropriate selecting adjacent frame matching.

\section{Division and selection of sub blocks}

The motion vectors of sub blocks are calculated after dividing the video image. The size of the sub block not only affects the amount of calculation, but also affects the accuracy of motion estimation. The size of image, the number of sub blocks, and the richness of gray information should be considered when dividing the video image[8]. In this paper, the video image is divided into 25 sub blocks, as shown in Fig.4. 


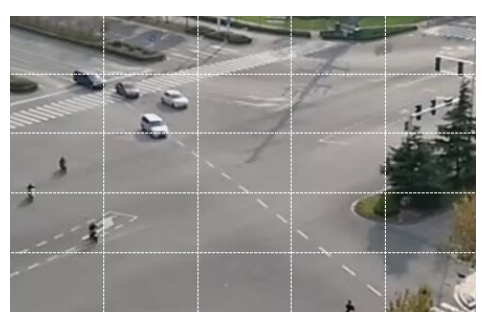

Fig.4 Division of sub blocks

Some sub blocks exist moving objects, which will affect the estimation of local motion vectors. These sub blocks should accordingly be removed. In this paper, the moving objects are detected by using the difference method of two adjacent frames. The calculation of the inter-frame difference is as follows.

$$
\begin{aligned}
& D_{n}(i, j)=\left|G_{n}(i, j)-G_{n-1}(i, j)\right| \\
& R_{n}(i, j)=\left\{\begin{array}{lll}
0 & \text { if } & D_{n}(i, j)>T \\
1 & \text { if } & D_{n}(i, j) \leq T
\end{array}\right.
\end{aligned}
$$

In two formulas, $G_{n}(i, j)$ and $G_{n-1}(i, j)$ are respectively the pixel values of two adjacent frames in coordinates $(i, j) ; D_{n}(i, j)$ is a difference image; $R_{n}(i, j)$ is a binarization image after the difference; $\mathrm{T}$ is the set threshold.

If there is a large number of moving objects in a certain sub block, the sub block should be removed. Fig. 5 is the image after the selection.

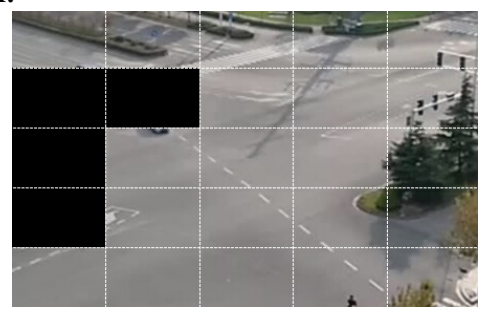

Fig. 5 Selection of sub blocks

\section{Global motion estimation}

Multiple local motion vectors of sub blocks are obtained after the calculation. Most of the local motion vectors can reflect the global motion of the image because there are few complex motions such as rotation now. So median filtering method is used to estimate the global motion. The local motion vector is sorted, and the resulting intermediate vector can be regarded as the global motion vector. This method avoids the complicated mathematical operations of other method like random sample consensus (RANSAC), which improves the real-time requirement of image stabilization.

\section{Results and analysis of experiments}

The experimental hardware platform includes the laptop computer of Intel Core $15 / 2.6 \mathrm{GHz}$, the camera, MSP430, MPU6050, stepping motors, etc. Some experiments are carried out with a real traffic video. The frame rate is $15 \mathrm{frame} / \mathrm{s}$, and the resolving power is $720 \times 640$. On one hand, the mechanical image stabilization based on MPU6050 is used to compensate the large deflection of the camera. On the other hand, the electronic image stabilization based on sub-block gray projection is used to stabilize the real-time video by VS2010 and OpenCV. The image stabilization region is $640 \times 480$ in the center.

Fig. 6 is the original video sequence in 5th, 20th, 35th, 50th, 65th, 80th frames. It can be found that there are some large or small offsets of the images. Fig.7 is the corresponding sequence after compound image stabilization processing in this paper. It turns out that the previous offsets are eliminated and the compound method can play a good stabilization effect. 


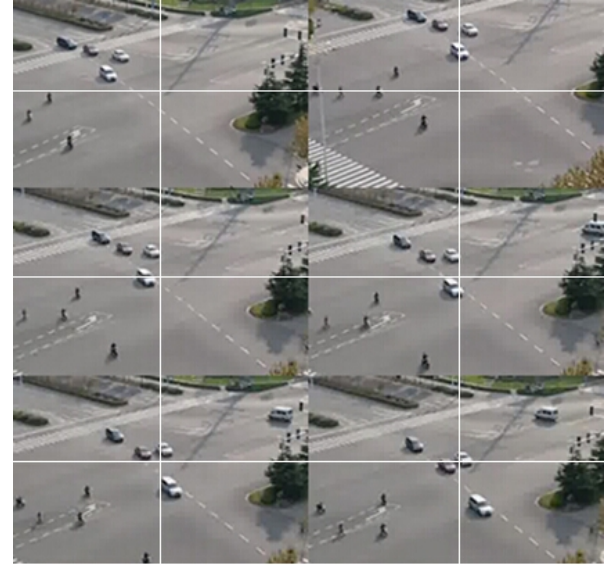

Fig.6 Original video sequence

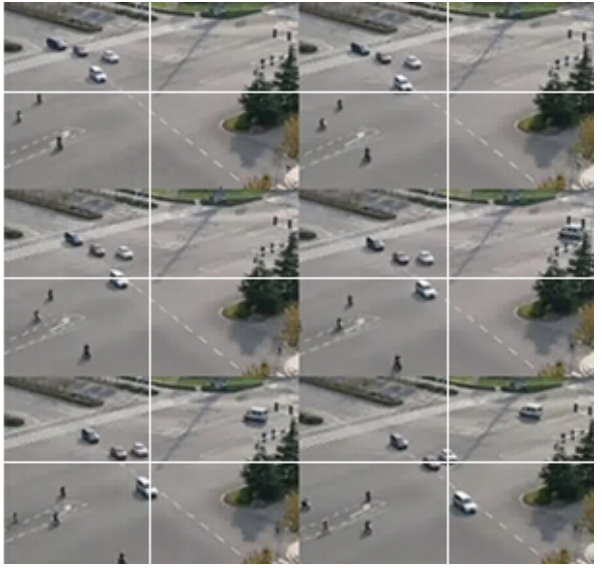

Fig.7 Corresponding sequence after processing

The evaluation of stabilization performance is also an important part of image stabilization technology. In addition to the subjective evaluation of the human eye, the commonly used objective index is peak signal to noise ratio (PSNR)[13].

In this paper, the PSNR of the adjacent frames is defined as follows.

$$
\operatorname{PSNR}\left(G_{n}, G_{n-1}\right)=10 \log \frac{255^{2}}{\operatorname{MSE}\left(G_{n}, G_{n-1}\right)}
$$

In Eq.9, $\operatorname{MSE}\left(G_{n}, G_{n-1}\right)$ is mean square error between the adjacent frames. Its definition is as follows.

$$
\operatorname{MSE}\left(G_{n}, G_{n-1}\right)=\frac{1}{N \times \mathrm{M}} \sum_{j=1}^{N} \sum_{i=1}^{M}\left[G_{n}(i, j)-G_{n-1}(i, j)\right]^{2}
$$

The bigger PSNR value is, the better the image stabilization effect is. In this paper, the only electronic image stabilization is compared with the compound image stabilization.

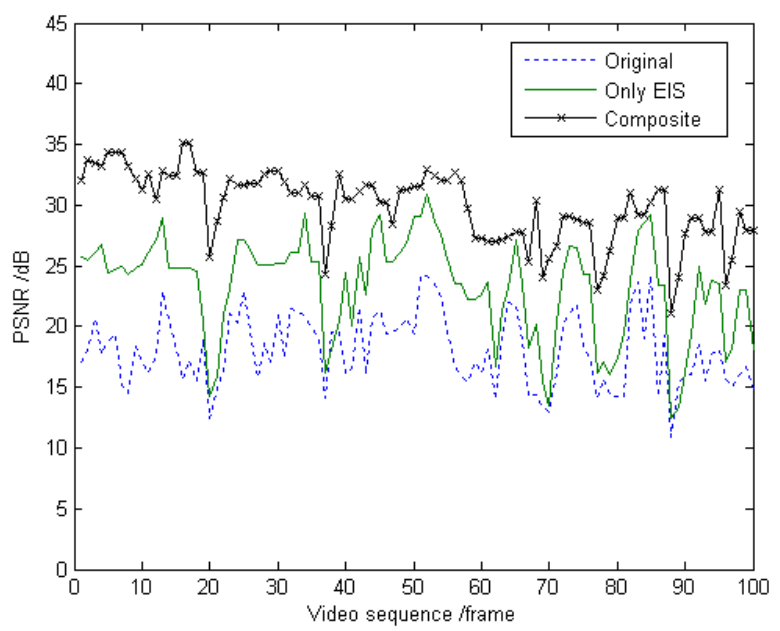

Fig. 8 Comparison of PSNR values

Fig.8 is a comparison of the PSNR of the original video sequence and the sequences after two image stabilization methods. When it comes to the large change of two frames, the only electronic image stabilization is very difficult to get the obvious effect. In comparison, the effect of the compound image stabilization is relatively stable. After using the only electronic image stabilization, the average PSNR of each frame is raised about $8 \mathrm{~dB}$. But after using the compound image stabilization, the average PSNR is raised about $16 \mathrm{~dB}$.

\section{Summary}

In this paper, MPU6050 is used to measure the deflection of the camera, and MSP430 controls stepping motors to eliminate the large angle. At the same time, the electronic image stabilization of the sub-block gray projection is used to stabilize real-time traffic video. So the compound image 
stabilization comes true. The experimental results show that the method can not only stabilize the large angle deflection, but also can effectively inhibit the interference of local moving objects. And the average PSNR is is increased by about $16 \mathrm{~dB}$ after the processing of the method.

\section{Acknowledgements}

This work was supported by the National Natural Science Foundation of China (61273024), the application basic research project funded by Ministry of transportation (2014319813180), the project funded by Jiangsu Education Department (14KJB510030), and the Natural Science Foundation Project of Nantong University (13ZJ003).

\section{References}

[1] Wang Zhimin,Xu Xiaogang. A survey on electronic image stabilization[J]. Journal of Image and Graphics,2010,15(3):470-480.

[2] Wang Zhen,Gao Fengqi,Gao Min. The development and application of new-type stabilization platform in infrared imaging guidance[J]. Aerodynamic Missile Journal,2015,03:77-80.

[3] Ma Zhiqiang, Chen Guangju,Shan Yong, et al. An overview on electronic image stabilization of the airborne system[J]. Electronic Design Engineering,2012,20:171-175.

[4] ZOKAI S, WOLBERG G. Image registration using Log-Polar mappings for recovery of large-scale similarity and projective transformations[J]. Image Processing, IEEE Transactions on ,2005,14(10): 1422-1434.

[5] HSIEH S P,KAO C H. A study of the feature-based digital image stabilization system[J]. Journal of the Chinese Institute of Engineers,2010, 33(4):635-641.

[6] Zhang Kun,Xu Tingfa,Wang Ping, et al. Real-time full-frame digital image stabilization system by SURF[J]. Optics and Precision Engineering,2011,08:1964-1972.

[7] Di Xiaoguang,Jin Wanxin,Yu Ying. Digital video stabilization for large-scale moving foreground object and rotation jitter[J]. Optics and Precision Engineering,2014,01:177-185.

[8] Wu Hao,Deng Hongbin. A video stabilization method based on sub-block gray projection[J]. Acta Armamentarii,2013,04:406-411.

[9] Invensense. MPU-6000 and MPU-6050 Product Specification Revision 3.3[R]. Sunnyvale: Invensense, 2012.

[10] Haque M N, Biswas M, Pickering M R, et al. A low-complexity image registration algorithm for global motion estimation[J]. Circuits and Systems for Video Technology, IEEE Transactions on, 2012, 22(3): 426-433.

[11] ZHAO Z, Chen Y. A fast electronic image stabilization algorithm of video sequences based on gray projection and block matching[J]. Opto-Electronic Engineering,2011,6:028.

[12] Ren Hang,Zhang Tao. Imaging CCD translation compensation method based on movement estimation of gradation projection technology[J]. Journal of Applied Optics,2009,03:417-422.

[13] Huang Chen, Wang Jian-jun, Gao Xin, et al. Study on video stabilization performance assessment in electronic image stabilization[J]. Laser and Infrared,2013,05:477-481. 